\title{
Sobrevivencia y producción de biomasa, de acacia pennatula (schlecht.) Benth por efecto de tres alturas de poda EE. 2012
}

\author{
Josué Tomás Urrutia Rodríguez ${ }^{1}$ \\ Oscar Rafael Lanuza ${ }^{2}$ \\ Alejandrina Herrera Herrera ${ }^{3}$
}

\section{RESUMEN}

En este ensayo se evaluó el rendimiento de biomasa, capacidad de rebrote, sobrevivencia de Acacia pennatula establecida como regeneración natural hace 10 años, Haciendo uso de tres alturas de podas (2, 3 y $4 \mathrm{~m}$ ), utilizando tres parcelas de $10 \mathrm{~m} 2$ en donde se tomaron ocho por cada parcela. Se realizó un corte inicial en Noviembre del 2012.

Se determino la biomasa leñosa y comestible. Con los resultados obtenidos se puede inferir que la producción de biomasa se ve levemente aumentada cuando las podas se realizan a 2 metros de altura con (578 kg de MS/ha), no presentando diferencias estadísticas comparadas con las demás alturas de poda $(\mathrm{P}>0.4993)$. La sobrevivencia de Acacia pennatula se mantiene en $100 \%$ cuando las podas se hacen a 4 metros de altura, no así cuando se hace a 2 o $3 \mathrm{~m}$ de altura. La capacidad de rebrote de la especies se ve estimulada cuando la poda se hace a cuatro metros llegando a un promedio de 101 rebrotes, al contario cuando se hace a 2 o 3 metros, la cantidad de rebrote disminuye en un 50\%.

La calidad de los rebrotes (longitud y diámetro basal) se ve estimulada se presentan como significativos $(\mathrm{P}<0.040)$, cuando se hacen las podas a menos alturas. Pero el incremento tanto de diámetro como de longitud de los rebrotes se ven incrementado cuando las alturas de podas se dan cuatro metros de altura, Se concluye que a mayor altura de poda permite aumento en la sobrevivencia y número de rebrotes, con una menor longitud y diámetros de los mismos. Pero con incrementos significativos. Se tendría que proseguir con las mediciones de producción de biomasa de los rebrotes obtenidos.

Palabras Claves: Acacia pennatula, altura de poda, sobrevivencia, rebrote

1 UNAN-Mangua/FAREM-Estelí, Estación experimental para el estudio del Trópico Seco. Correo electronico: josuerod20@yahoo.com 2 UNAN-Mangua/FAREM-Estelí, Estación experimental para el estudio del Trópico Seco. Correo electronico: rafa.artesano@gmail.com 3 UNAN-Mangua/FAREM-Estelí, Estación experimental para el estudio del Trópico Seco. Correo electronico: alejandjes@gmail.com 


\title{
Survival and biomass production, of Acacia pennatula (schlecht.) Benth for effect of three pruning heights EE. 2012
}

\author{
Josué Tomás Urrutia Rodríguez ${ }^{1}$ \\ Oscar Rafael Lanuza ${ }^{2}$ \\ Alejandrina Herrera Herrera ${ }^{3}$
}

\begin{abstract}
In this study, the biomass yielding was evaluated, regrowth capacity, and survival of Acacia pennatula established as natural regeneration 10 years ago. Making use of three pruning heights (2, 3 and $4 \mathrm{~m})$, using three $10 \mathrm{~m} 2$ plots in which eight were taken per each plot. An initial cut was made in November 2012 and edible woody biomass was determined.

With the results obtained it can be inferred that biomass production increases slightly when pruning is performed at a 2 meter high ( $578 \mathrm{~kg} \mathrm{DM} / \mathrm{ha}$ ), with no statistical differences compared to other cutting heights $(\mathrm{P}>0.4993$. The survival of Acacia pennatula remains at $100 \%$ when pruning is done at 4 meters high, but not when it is done at a 2 or $3 \mathrm{~m}$ high. The regrowth capacity of the species is encouraged when pruning is at four meters reaching an average of 101 regrows, contrary to when it is done within 2 or 3 meters, the amount of regrowth decreases by $50 \%$.
\end{abstract}

The quality of the regrows (length and basal diameter) is stimulated and it is presented as significant $(\mathrm{P}<0.040)$, when pruning is done in reduced heights. But the increase in both diameter and length of the regrows are increased when the heights of pruning are given at four meters high. It is concluded that the pruning height allows the increase in survival and in number of regrows, with a shorter length and diameters thereof; but with significant increases. Measurements of the production of biomass of regrows obtained would have to be continued.

Keywords: Acacia pennatula, pruning height, survival, regrowth.

1 UNAN-Mangua/FAREM-Estelí, Experimental Station for the study of the Dry Tropics. E-mail: josuerod20@yahoo.com 2 UNAN-Managua/FAREM-Estelí Experimental Station for the study of the Dry Tropics. E-mail: rafa.artesano@gmail.com 3 UNAN-Managua/FAREM-Estelí Experimental Station for the study of the Dry Tropics. E-mail: alejandjes@gmail.com 


\section{INTRODUCCIÓN}

En Nicaragua muchas de las especies forestales son de gran importancia como leña o combustible para la cocción de alimentos en los sectores rurales y urbanos. Siendo el sector rural el que depende exclusivamente de la madera como fuente de combustibles. Lo que produce una gran presión sobre el recurso bosque y forestal (Gregersen et al 1994; Nieto H. 2000).

Tomando en cuenta lo anterior y según (MAGFOR, 2009) la especie carbón, es una de las 60 especies, indicadas por especialistas forestales de nuestro país, como especie potencialmente comercial por sus múltiples usos en especial por el uso de ella como energética, maderable (Postes, arados), rápido crecimiento y por su importancia como forrajera en la época más crítica, como lo es la periodo seco del año. Otra de la habilidades del A. pennatula según el (OFICATIE, 2000) hace notar la importancia de manejar el carbón mediante cortas repetidas por su fuerte habilidad para el rebrote.

Acacia pennatula es capaz de colonizar y persistir tras la transformación del bosque seco tropical a la vez, una especies que se ha vuelto muy común en bosques secos transformados de toda Mesoamérica (Peguero, G. 2012). Según nieto (Nieto, H. 2000) es el árbol más utilizado en la Paisaje Terrestre Protegido Miraflor Moropotente, por la población de este lugar con un $33 \%$ de 28 especies utilizadas en la reserva. En la actualidad dados los usos posibles de la especies Acacia penntula, se está utilizando mas para forraje que para leña. En cuanto al uso como leña ha disminuido 30\%, comparándolo con el uso que se le daba anteriormente para esta función. Entre los usos que sobresalen del carbón tenemos que son piezas importantes para postes y postes maestros, pero también para la elaboración de arado.

Es por esto que los cambios drásticos en el régimen de perturbación como en el conjunto de interacciones bióticas, una gran parte de las especies forestales características del bosque seco mesoamericano no consiguen ni persistir ni propagarse, mientras que hay otras cuya abundancia y distribución se ha visto altamente favorecida (Esquivel et al., Tarrasón et al., 2010). Y se puede llevar a mención el caso de Acasia pennatula Benth., una leguminosa mimosoidea común como árbol disperso en potreros y en bosques secundarios desde México hasta el Ecuador (Ebinger et al 2000).

En cuanto a su capacidad como plantas nucleadoras se deben de hacer estudios más a profundidad dado que si se pueden observar en campo esta capacidad de nuclear a especies con similares capacidades como especie colonizadora y donde se pueden observar creciendo diferentes especies debajo de sus ramas a muy cerca de sus tallos y raíces. Slocum (2001) afirma que las especies pioneras capaces de establecer individuos aislados en los potreros, tendrían una importancia capital como "Nucleadoras" de la sucesión al facilitar el establecimiento de plántulas de otras especies tras el abandono de uso ganadero. Teniendo en cuanta siempre que pueden existir especies capaces de establecerse y pueden bloquear la sucesión durante décadas (Burgos \& Maass 2004; Álvarez-Yépiz et al,. 2004; Peguero, G. 2012).

Al mismo tiempo como beneficio directo lo más recomendables financieramente es la utilización y producción de árboles de carbón, como postes con turnos de 19 años, por su mayor valor actualizado por volumen y unidad de tiempo (Nieto, H. 2000).

Por la importancia ecológica y socioeconómica que posee y brinda la especie Acacia pennatula, y tomando en cuenta la presencia en un 70\% del área en la Estación Experimental en la FAREM-Estelí, se evaluara la capacidad de esta especie del rebrote bajo diferentes intensidades de podas y composición tanto del banco de semillas bajo del carbón como de los brízales y latizales 
La presencia de esta especie y su dominancia en el territorio, que compone la Estación nos permite y nos empuja a realizar algunos trabajos de investigación que nos indiquen cual es el manejo más adecuado que se le debe dar a la especie lo que permitirá tener una herramienta que permita al productor saber cuánto está produciendo el bosque de Acacia pennatula y el manejo mas adecuado que se le debe de dar, a este tipo de vegetación.

\section{MATERIALES Y METODOS}

\section{Ubicación del área}

Localización y clima: Las Estación Experimental se encuentra ubicada dentro de la zona de amortiguamiento de Área Protegida de El Tisey - La Estanzuela, dicha Estación se encuentra a $1.5 \mathrm{~km}$ de al suroeste de la ciudad de Estelí. Este sector pertenece según Holdrich, al Bosque seco Tropical, con temperaturas promedio de $24-26^{\circ} \mathrm{C}$, con lluvias irregulares comprendida entre los meses de mayo y octubre, con un periodo canicular de julio a agosto, con una precipitación anual que oscila entre $800-900 \mathrm{~mm}$ y una altura de 800 y $884 \mathrm{msnm}$ (Zeledón, A. 2004). Su ubicación geográfica se encuentra entre las coordenadas 0568720 y 1443707.

Suelos: Los suelos del área de La Estación Experimental se clasifican como suelos vertisoles, de textura franco arcillosa, suelos muy compactados, aunque ricos en fosforo, potasio y calcio pero bajas cantidades de Materia Orgánica muy pobre en nitrógeno, presentado pH (C2O) cercanos a la neutralidad (Alcañiz, 2005; Archivos Estación Experimental). Dado el grado de degradación de los suelos se determina, dejar reposar el terreno para evitar que siguiera la compactación y erosión hídrica en los mismos. Es de esta manera que en este periodo de reposo permite la regeneración natural la cual en un $75 \%$ de la Estación está conformada por vegetación secundaria, la que es dominada por carbón (Acacia pennatula), que está ubicada en diferentes sectores de la Estación y que tiene diferentes edades que van desde los ocho, nueve y diez años (Urrutia, J 2007).

Diseño y Tratamiento: En la áreas en regeneración natural con dominancia de carbón (Acacia pennatula), en la Estación Experimental, que se estableció sobre un sustrato herbáceo dominado por zacate Estrella Cynodon nlenfuensis Vanderyst, se estudio la influencia de tres alturas de poda (2, 3 y $4 \mathrm{~m})$, en la producción de biomasa foliar, ramas delgadas y ramas gruesas, capacidad de rebrote, sobrevivencia. Se emplearon parcelas de $50 \times 50 \mathrm{~m}$, a lo interno de estas se establecieron sub-parcelas de $10 \times 10 \mathrm{~m}$, podando el total de los individuos pertenecientes a esta especie $\mathrm{y}$ tomando tres individuos para la realización de los pesos de las tres estructuras de la planta, para sus respectivas medicines.

Procedimiento experimental: Se dispondrán tres parcelas por cada uno de los sectores de bosques de (Acacia pennatula), de las cuales se dispondrán 25 subparcelas de $10 * 10 \mathrm{~m}$, donde se tomaran en cuenta todos los individuos pertenecientes a la especies. Se efectuó una poda inicial en noviembre de 2012, para establecer la distribución de los tratamientos (altura de corte) a las unidades experimentales cada uno de los árboles de corte (árboles) ubicado en cada parcela y se definió la sobrevivencia de los individuos sometido a la poda. Con el fin de conocer como se comportara la masa residual de los rebrotes luego de la intervención, se utilizara los incrementos porcentuales que reflejen cuales son las ganancias en diámetro basa y longitud del cada rebrote por tratamiento. Se utilizara la ecuación recomendada por (Husch et al 1982) $\mathbf{I}(\mathbf{x})=(\mathbf{M} 2-\mathbf{M 1}) / \mathbf{M 1}$

Mediciones: En el primer momento de midieron las alturas de la base al ápice del individuos y diámetros a la altura del pecho, de las unidades experimentales dentro de las parcelas. Se realizaron mediciones cuantitativas de la biomasa foliar (hojas y retoños no lignificados) y de la biomasa leñosa (ramitas lignificadas y ramas gruesas); a continuación se secaron las muestras en 
la estufa a $70^{\circ} \mathrm{C}$, por un periodo de 48 horas, para determinar la biomasa seca. Al mismo tiempo se realizaron los conteos con los cuales se determino la sobrevivencia por cada una de las alturas de poda.

Con las mediciones realizadas anteriormente se encontraron los rendimientos en biomasa por hectárea a diferentes alturas de poda, capacidad de rebrote $y$ sobrevivencia de los individuos sometidos a poda.

A partir de la poda realizada y datos obtenidos en la evaluación de biomasa, rebrote y sobrevivencia se relazaron análisis de la varianza para demostrar la significancia de los indicadores medidos bajo las diferentes podas realizadas a los individuos de carbón. Para el análisis de los datos se utilizará el programa StatView.

\section{RESULTADOS}

\section{Determinar la producción de biomasa en arboles de Acacia pennatula}

Los rendimientos de biomasa total, leñosa y comestible cosechada a la primer corta no difieren estadísticamente entre los tratamientos aplicados $(\mathrm{P}>0.4993)$.

Los mejores resultado al evaluar la biomasa se encuentra que las alturas de poda que presentan mayores kilogramos de biomasa estas cuando se podan los árboles a 200 y $400 \mathrm{~cm}$, presentado los menores rendimiento en biomasa seca la poda a $300 \mathrm{~cm}$, lo cual puede estar dado por la posición de los arboles dentro del bosque.

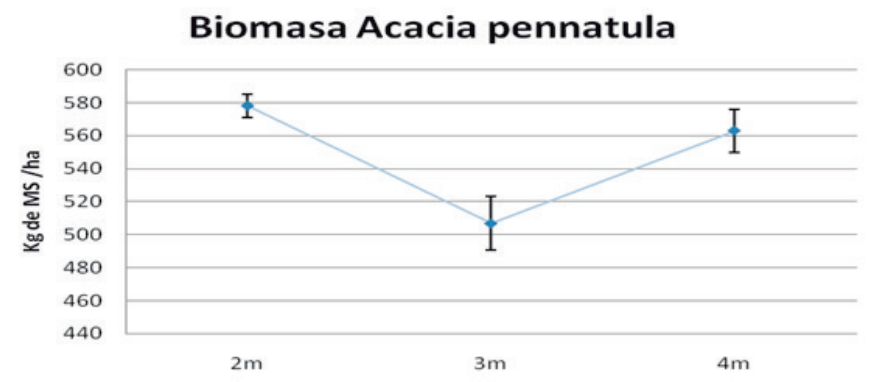

Gráfica 1. Biomasa de Acacia pennatula (EE 2013)
Estos resultados pueden estar dado por la edad que presentan los arboles dado que es similar en estos carbonales que puede estar por los 10 años de edad y están en la misma altura además de ser coetáneos.

El incremento a los 200 y $400 \mathrm{~cm}$ posiblemente esté relacionado con el incremento de los recursos ambientales (agua, temperatura y luminosidad) y el uso más eficiente de los nutrientes por algunas individuos; esto motiva una menor movilización de los carbohidratos solubles y otras reservas como una única fuente de producción vegetativa (Soca et al 1997; Mochiutti, 1995).

Tabla 1. Producción de biomasa total y por cada uno de las estructuras evaluadas

\begin{tabular}{|c|c|c|c|}
\hline $\begin{array}{c}\text { Altura de } \\
\text { corte cm }\end{array}$ & $\begin{array}{c}\text { Biomasa } \\
\text { Total kg } \\
\text { de MS/ha }\end{array}$ & $\begin{array}{c}\text { Biomasa } \\
\text { Comestible } \\
\text { kg de MS/ha }\end{array}$ & $\begin{array}{c}\text { Biomasa } \\
\text { leñosa kg } \\
\text { de Ms/ha }\end{array}$ \\
\hline $2 \mathrm{~m}$ & 578 & 140 & 438 \\
\hline $3 \mathrm{~m}$ & 507 & 146 & 361 \\
\hline $4 \mathrm{~m}$ & 563 & 141 & 422 \\
\hline
\end{tabular}

\section{Efecto de la intensidad de poda en arboles de Acacia pennatula}

La poda aplicada a diferentes grupos de árboles de Acacia pennatula fue realizada en tres fechas, los efectos de la poda se demuestran gráfica 2 en donde los árboles con mas individuos sobrevivientes es la poda aplicada a 4 metros de altura con una sobre vivencia hasta el momento de la medición del 100\%, lo cual por el tipo de poda, se espera que valla muriendo individuos al pasar del tiempo, aunque algunos autores indican que las especies arbóreas propias del bosque seco tiene alta capacidad de rebrote (Mostacedo, 2001) y que esta capacidad la tienen el $45 \%$ de las especies arbóreas.

Los tratamientos con podas a menos de 4 metros de alturas presentan una sobrevivencia menor al 75\% de 
los individuos. A pesar de la alta capacidad de rebrote de las especies arbóreas existen otras variables que se tiene que tener en cuenta, tales como el momento de la poda, el esta fisiológico de la planta, las plagas fitófagas que estén relacionadas con las especies arbóreas del lugar.

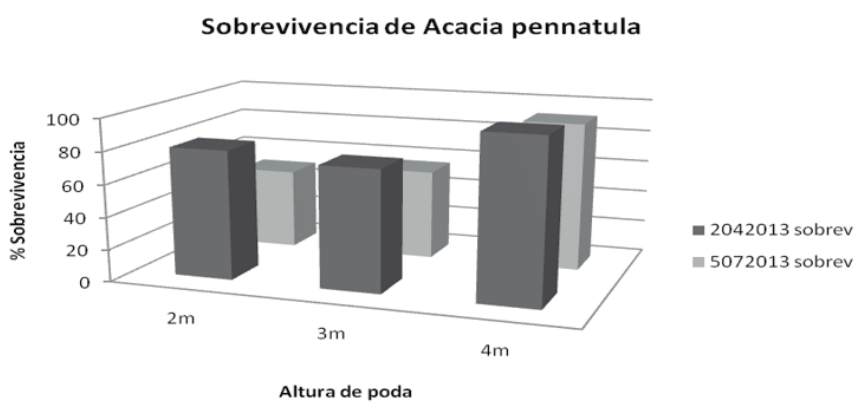

Grafica 2: Sobrevivencia de arboles de Acacia pennatula a diferentes de poda (2013)

Se debe de tener en cuenta que la capacidad de rebrote de las especies arbóreas propias del bosque seco tropical pueden ver afectada su capacidad de rebrote después de una perturbación intensas y más si estas ocurren hacia el final de la estación seca, lo que según Peguero (2012) podría estar dado por la reducción de la disponibilidad de algunos elementos como el $\mathrm{N}$ y $\mathrm{P}$, cuando se provocan alteraciones sobre los árboles. Otros autores han observado en Glericidia sepium, que las cantidades de almidón y azucares solubles después de las poda disminuyen, principalmente en las reservas del tallo, a cambio especies como Leucaena leucocephala, las reserva tanto en tallo como en raíces para restaurar el total de las reservas después de la poda (Casanova-Lugo, et al 2010; Latt etal., 2000).

Se puede observar el ataque de algunos escarabajos que raspan la corteza de los arboles podados, ovopositando sus huevos por todo el contorno del tallo. MacíasSámano (2010) no dice que la podas no debe de exceder más de una cuarta parte del árbol, ya que el no seguir esta regla, el vigor del árbol disminuiría fuertemente, promoviendo el desbalance entre el crecimiento de la copa y la raíz, el árbol puede estresarse, y pude ser presa fácil de enfermedades y algunos insectos barrenadores.
Lo anterior explica la muerte paulatina de los arboles que se cortaron por debajo de los tres metros.

\section{Capacidad y calidad de los rebrote encontrados en Acacia pennatula}

La capacidad de rebrote en Acacia pennatula se ve afectada por la altura de poda como se puede notar cuando se hace a mayor altura la poda, mayor es la cantidad de rebrotes que se dan en la poda realizada a 4 $\mathrm{m}$ de altura produce en promedio hasta 101.3 rebrotes, al contrario las podas realizadas más baja en el fuste de la planta, se presentan como menores en la cantidad rebrotes presentando en promedio los tratamientos 2 y $3 \mathrm{~m} 59$ y 52 rebrotes un poco más de la mitad de los que presentan la poda a realizado a cuatro metros.

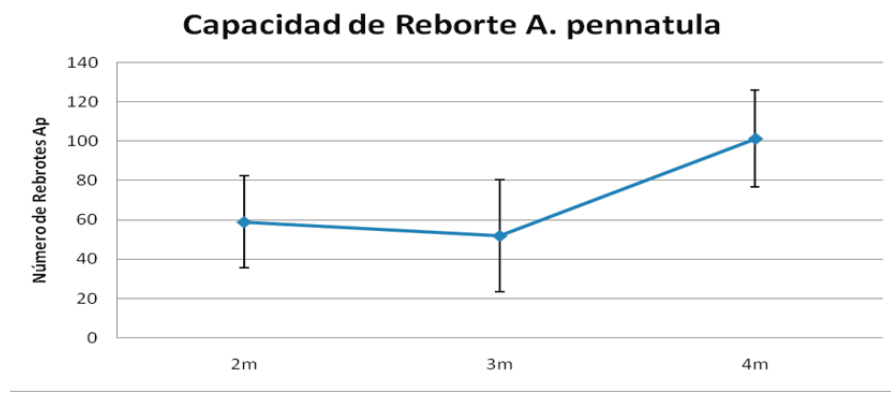

Grafica 3. Capacidad de rebrote a diferentes alturas de poda en A. pennatula, (EE 2013).

Este hallazgo se puede explicar en algunas investigaciones realizadas con antelación por otros investigadores que afirman que la altura de podas al ser más elevadas podría activar las producciones de biomasa (rebrotes) (Soca et al 1997), Field y OeMatan (1990) y Gizachew (1992) obtuvieron rendimientos más significativos con la mayor altura empleada. También se han observado incrementos en la producción al realizar cortes a mayores alturas (Gutteridge y Shelton, 1993). 
Tabla 2. Calidad de los rebrotes obtenidos por cada una de las estructuras evaluadas a diferentes alturas en A. pennatula.

\begin{tabular}{|l|r|r|r|}
\hline Altura de poda (m) & 2 metros & 3 metros & 4metros \\
\hline Diametro $(\mathrm{mm})$ & 0.31 & 0.43 & 0.26 \\
\hline ES+- & 0.032 & $0.52 *$ & 0.025 \\
\hline $\begin{array}{l}\text { Longitud Rebrote } \\
(\mathrm{cm})\end{array}$ & 37 & 33.6 & 14.5 \\
\hline ES+- & $7.2 * *$ & $4.1 * *$ & 2.1 \\
\hline
\end{tabular}

En cuanto al diámetro de los rebrotes podemos notar que los emitidos después del tratamiento de poda a tres metros se presentan como los más largos dentro de los tratamientos evaluados y son estadísticamente mayores $(\mathrm{P}<0.040)$, lo cual puede estar explicado en que estos árboles son de diámetros mayores y hayan tenido una mayor exposición a la radiación solar.

Al igual al medir la longitud de los rebrotes en los tratamientos en los que se aplico una poda a 2 y 3 metros, de altura se presentan como estadísticamente significativos o son los más largos comparados el tratamiento aplicado a 4 metros de altura (tabla 2)

\section{Incremento de crecimiento de rebrotes}

Chaves \& Mora 2010; Johnston et al 1967 recomiendan estos tipos de estudios dado que permiten determinar los rendimientos en las diferentes edades de las plantas.

\section{CONCLUSIONES}

La poda recomendada en árboles de Acacia pennatula tiene una mayor sobrevivencia cuando se hacen las podas a 4 metros de altura, dado que presenta hasta un 100\% de sobrevivencia de los individuos podados.

La producción de biomasa (MS) no presenta diferencias significativas, haciendo la poda a diferentes alturas la biomasa total, leñosa y comestible es similar en los tres tratamientos aplicados.
La poda se realiza a $4 \mathrm{~m}$ produce una mayor presencia de rebrotes, hasta 101.1 rebrotes, lo cual está dado por la altura de poda y el menor ataque de insectos sobre los arboles muestreado.

En los individuos podados a 2 y 3 metros de altura por tener una menor cantidad de rebrotes, se ve estimulado la longitud y diámetro (dab) de los mismos, y se presentan como altamente significativos, al comparar los diferentes tratamientos.

\section{BLIBLIOGRAFIA}

González, L. 2010. Evaluación de los cambios ecológicos en la estructura vegetal de las pasturas en la macrozona semiseca de Miaraflor Moropotente. Tesis mografica. 52p.

Peguero, G. 2012. Colonización y persistencia de Acacia pennatula en bsoques tropicales secos transformados. Tesis Doctoral. Bellaterra, Barcelona. 144p.

López et al. 2003. Realciones alométricas para la predicción de biomasa forrajera y leña de Acacia pennatula y Guazuma ulmifolia en dos comunidades del norte de Chiapas, México. INCI vol.28 no.6 Caracas junio 2003. Asociación Interamericana. $15 \mathrm{p}$.

Macías-Sánano. 2010. Manual de podas para árboles. Con énfasis en el uso de podas para el contro del barrenador Hypsipyla grandella, plaga del Cedro y la Caoba. ECOSUR, Colegio de la Frontera Sur. $26 \mathrm{p}$.

Nieto, Hernández. 2000. Contribucion de la Acassia pennatula (Carbón) a la productividad agroforestal sostenible de la Reserva Natural Protegida Miraflor Moropotente. Thesis $N^{\circ} 677$. C.2. 69p.

MAGFOR, 2009. Resultados del inventario nacional forestal Nicaragua 2007-2008. 229p.

Ruiz, S. 2010. Lluvia de semillas de Lupinus elegans Kunth en un proyectos de restauración ecológica. Biológicas. 12(2):72-74. 3pag.

Urrutia, J. 2007. Flora y Fauna en el Centro de 
Investigación y Educación Ambiental. Revista OSTAYO. FAREM-Estelí, UNAN-Managua. 24p. Zeledón, A. 2004. Estructura del Bosque seco Tropical y sus usos en las comunidades El Limón, El Coyolito y El Dorado. Tesis de Maestría Medio Ambiente y Recursos Naturales. 125p.

Ebinger, J.E., Seigler, D.S., \& Clarke, H.D. (2000) Taxonomic revision of South American species of the genus Acacia subgenus Acacia (Fabacea: Mimosoideae). Systematic Botany, 25, 588-617.

Esquivel, M.J., Harvey, C.A., Fienegan, B., Casanoves, F., \& Skarpe, C. (2008) Effects of pastures managament on the natural regeneration of neotropical tree. Jurnal of Applied Ecology, 45,371-380.

Slocum, M.G. (2010) How tree spcies differ as recruitment foci in a tropical pasture. Ecolgy, 82, 2547-2559.
Mostacedo, B. et al. (2001) Regeneración y Silvicultura de Bosques Tropicales en Bolivia. Ecología de Semillas y plántulas de arboles maderables en bosques tropicales de Bolivia. Santa Cruz, Bolivia. 224p.

Medina, E. Pruning Mature Trees, de la International Society of Arboriculture, Champaign, IL, por José Eduardo Medina, Arbolista Certificado, Chicago, IL. Sociedad Internacional de Arboricultura (ISA). http://www.isahispana.com/treecare/resources/ pruning_mature_trees.pdf.

Casanova-Lugo et al. 2010. Efecto del intervalo de poda sobre la biomasa foliar y radical en árboles forrajeros en monocultivo y asociados. Tropical and Subtropical Agroecosystems, vol. 12, núm. 3, setiembre-diciembre, 2010, pp. 657-665,

Universidad Autónoma de Yucatan México. Red de revistas de América Latina, Caribe, España y Portugal. Sistema de Información Cientifica. 